\title{
A-17-2020 \\ SISTEMAS DE CULTIVO EN LIMEROS: EFECTOS EN LA PRODUCCIÓN Y CRECIMIENTO
}

\author{
Mira-García, A.B.(P), Conejero, W., Vera, J., Ruiz-Sánchez, M.C.
}

Departamento de Riego. CEBAS-CSIC. Campus Universitario de Espinardo, Apartado 164, 30100 Murcia, España.

abmira@cebas.csic.es; wenceslao@cebas.csic.es; jvera@cebas.csic.es; mcruiz@cebas.csic.es

\section{Resumen}

El objetivo principal del trabajo es el de evaluar los efectos de diferentes sistemas de cultivo: al aire libre (en meseta, sin meseta) y bajo malla de sombreo, en la producción y el crecimiento vegetativo de limeros (Citrus latifolia Tan., cv. Bearss) jóvenes. El ensayo se llevó a cabo durante dos campañas (2018 y 2019) en la finca experimental del CEBAS-CSIC en Murcia. El crecimiento vegetativo se evaluó con medidas del diámetro del tronco y del ancho y alto de copa, y el crecimiento de fruto con medidas de su diámetro ecuatorial. En el momento de la recolección se determinaron los kilos de fruta y número de frutos por árbol y se calculó el peso unitario medio del fruto. Los resultados obtenidos apuntan a que los árboles cultivados bajo malla de sombreo mostraron un mayor volumen de copa que los cultivados al aire libre, debido a una significativa mayor altura. La producción fue también mayor (aunque no significativa) en los árboles bajo sombreo, debido a un mayor tamaño de las limas durante la última fase de su crecimiento. La disminución de la radiación incidente en las condiciones de sombreo, propiciaron la creación de un microclima más favorable para la asimilación de carbono, lo que parece provocar unas mejores condiciones para el desarrollo vegetativo de los árboles y la producción de limas de calidad.

\section{1) Introducción}

España es el productor de cítricos más importante de la Unión Europea, con 7528 Mt, y el primer exportador en el mundo (4,1 Mt) (FAO, 2017). En el territorio nacional, la producción citrícola se concentra en la Comunidad Valenciana (4,1 Mt), Andalucía (2,3 Mt) y la Región de Murcia (1 Mt) (MAPA, 2019). En la Región de Murcia tradicionalmente la especie por excelencia es el limón, representando el $64 \%$ de la producción citrícola total (CARM, 2018), aunque en los últimos años otras especies cítricas como la lima están diversificando la oferta citrícola de la región. Prueba de ello es que en los últimos 5 años la producción de lima ha aumentado en más de un 25\% (CREM, 2019) convirtiendo este cultivo en una potencial alternativa a los cultivos cítricos tradicionales.

La elección del sistema de cultivo en nuevas plantaciones es un factor clave que condicionará todas las operaciones culturales futuras y que puede afectar al crecimiento vegetativo, así como a la producción (Manja and Aoun, 2019; Mditshwa et al., 2019). El cultivo bajo malla comúnmente se orienta a sombrear y proteger los árboles ante situaciones de estrés abiótico (altas temperaturas, excesiva radiación, etc.), o de cualquier inclemencia meteorológica. A su vez, los cambios en el microclima producidos por el sombreado pueden inducir modificaciones en el comportamiento fisiológico de la planta. En varios estudios se han observado aumentos en la conductancia estomática y en la asimilación de $\mathrm{CO}_{2}$ (Medina et al., 2002), así como en la capacidad fotosintética de las plantas cultivadas bajo malla de sombreo (Incesu et al., 2016; Zhou et al., 2018). Estas modificaciones en el comportamiento fisiológico se traducen en cambios en la eficiencia en el uso del agua (Alarcón et al., 2006) 
afectando al crecimiento vegetativo (García-Sánchez et al., 2015), así como a la producción y el rendimiento del cultivo (Wachsmann et al., 2014).

\section{2) Objetivo}

El objetivo principal del trabajo es evaluar el efecto de diferentes sistemas de cultivo: aire libre (cultivo en meseta, sin meseta) y con malla de sombreo, sobre la producción y el crecimiento vegetativo de limeros jóvenes regados por goteo en las condiciones mediterráneas del sureste español.

\section{3) Material y Métodos}

El ensayo se llevó a cabo en los años 2018 y 2019 en la finca experimental del CEBAS-CSIC en Santomera (Murcia), en una parcela de 1,2 ha. El suelo es pedregoso, altamente calcáreo ( $45 \%$ de carbonato cálcico), de textura franco-arcillosa (capacidad de campo y punto de marchitez 0.32 y $0.14 \mathrm{~m}^{3} \mathrm{~m}^{-3}$, respectivamente) y con un bajo contenido en materia orgánica. El material vegetal empleado fueron limeros (Citrus latifolia Tan., cV. Bearss) de 3 años de edad, injertados sobre Citrus macrophylla L., con un marco de plantación de $6 \mathrm{~m} \times 5 \mathrm{~m}$. El sistema de riego localizado consistió en una doble línea portagoteros, con 4 goteros autocompensantes de $4 \mathrm{~L} \mathrm{~h}^{-1}$ por árbol, situados equidistantes a 0,75 $\mathrm{m}$ del tronco. Las necesidades hídricas de la plantación se determinaron con un protocolo de riego automático basado en la reposición del stock hídrico del suelo, con un agotamiento permisible del 10\% (Vera et al., 2019). Para ello se emplearon sondas capacitivas (EnviroPro ${ }^{\circledR}$, Entelechy Pty. Ltd., Golden Grove, Australia) instaladas a 0,1 m del emisor más cercano al tronco, en 4 árboles. Las sondas permitían monitorizar en continuo el contenido volumétrico de agua en el perfil 0-0,8 m del suelo y actuar en la apertura/cierre de las electroválvulas. El sistema de telemetría tomaba lecturas cada 5 min y registraba valores cada $15 \mathrm{~min}$. Las unidades de radio-transmisión enviaban datos al servidor web addVANTAGE (ADCON Telemetry, Austria) para el procesamiento y la visualización de los datos.

Se establecieron tres sistemas de cultivo:

1) Meseta-aire libre (MESETA): los árboles estaban plantados en mesetas (2 $\mathrm{m}$ de ancho y $0,4 \mathrm{~m}$ de alto) al aire libre (Figura $1 \mathrm{~A}$ ).

2) Meseta-malla de sombreo (SOMBREO): los árboles estaban plantados en mesetas ( $2 \mathrm{~m}$ ancho y $0,4 \mathrm{~m}$ alto), dentro de un umbráculo de $4,5 \mathrm{~m}$ de altura, con techo de tipo zig-zag. La malla empleada era de polietileno de alta densidad, con un coeficiente de sombreo del $24 \%$ y una porosidad del $58,4 \%$ (Figura 1B).

3) Sin meseta-aire libre (SIN MESETA): los árboles estaban plantados directamente sobre el suelo al aire libre (Figura $1 \mathrm{C}$ ).

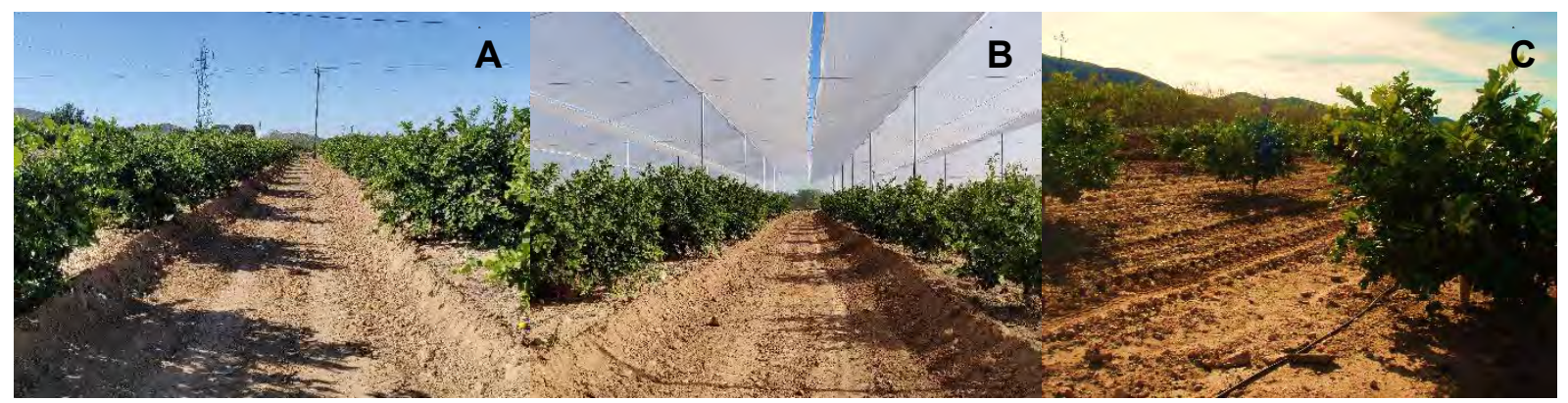

Figura 1. Vista general de limeros (Citrus latifolia Tan., $c v$. Bearss) jóvenes en los diferentes sistemas de cultivo: meseta-aire libre (A) meseta-malla de sombreo (B) y sin meseta-aire libre (C). 
Cada sistema de cultivo fue evaluado en parcelas independientes de $60 \mathrm{~m} \times 40 \mathrm{~m}$, que contaban con un total de 5 filas de árboles con 12 árboles por fila (60 árboles en total). Las medidas fueron tomadas en 8 árboles de las filas centrales de cada sistema de cultivo, el resto fueron considerados árboles bordes.

Durante los dos ciclos de cultivo, en los árboles objeto de estudio se realizaron medidas periódicas del crecimiento vegetativo: diámetro del tronco $\left(\varnothing_{\text {tronco }}\right)$ a $0.30 \mathrm{~m}$ del suelo, con un pie de rey (Codimex-C100cm, Canadá), longitud de brotes, con cinta métrica en brotes marcados y alto y ancho de copa, con jalones, determinando el volumen de la copa de los limeros (asimilado a un cilindro). Además, quincenalmente, en los mismos árboles se midió el diámetro ecuatorial del fruto, con un calibre digital (Mitutoyo, CD-15D), en 100 frutos elegidos al azar en cada sistema de cultivo ( 25 frutos árbol $^{-1}$ ). En el momento de la recolección (finales de agosto de 2018 y 2019) se determinó la producción en los 8 árboles testigo de cada uno de los sistemas de cultivo, evaluando la carga productiva (kg árbol $^{-1}$ ) con una balanza de precisión y el número de frutos por árbol, obteniéndose el peso unitario medio del fruto.

Para evaluar el estado hídrico de las plantas se realizaron medidas discretas (cada 10-15 días, durante el periodo experimental) del potencial hídrico de tallo ( $\left.\Psi_{\text {tallo }}\right)$ así como del intercambio gaseoso foliar: fotosíntesis neta $\left(F_{n}\right)$ y conductancia estomática $\left(g_{s}\right)$, en 1-2 hojas por árbol y en 4 árboles por sistema de cultivo. El $\Psi_{\text {tallo }}$ se midió a mediodía solar, con una cámara de presión (Soil Moisture Equipment Corp., Model 3000) en hojas sanas, maduras, localizadas en la cara norte del árbol y cerca del tronco. Estas hojas fueron tapadas al menos dos horas antes de la medida, siguiendo las recomendaciones de Hsiao (1990). El intercambio gaseoso se determinó con un medidor portátil de fotosíntesis (LI6400, Li-Cor, Lincoln, NE, USA) a primera hora de la mañana $(\approx 8: 00$ hora solar) en hojas maduras, soleadas situadas en la cara sur del árbol.

\section{4) Resultados y Discusión}

Las medidas de los indicadores del estado hídrico (potencial hídrico de tallo e intercambio gaseoso) presentaron valores que aseguraban un óptimo estado hídrico de los árboles $\left(\Psi_{\text {tallo }}>-0.9 \mathrm{MPa}, \mathrm{g}_{\mathrm{s}} \approx 50 \mathrm{mmol} \mathrm{m}^{-2} \mathrm{~s}^{-1}\right)$ durante todo el ensayo, en los tres sistemas de cultivo estudiados (datos no mostrados).

\section{1.) Crecimiento vegetativo}

La dinámica anual del crecimiento vegetativo de los limeros fue muy similar en los dos años y en todos los sistemas de cultivo estudiados, tanto para el diámetro del tronco, brotes (datos no mostrados), como para el volumen de copa (Figura 2). Es de destacar que las velocidades máximas de crecimiento tuvieron lugar tras la recolección de las limas (a finales de agosto) en ambos ciclos de cultivo. Este hecho demuestra la competencia por los foto-asimilados entre el crecimiento vegetativo y el del fruto (Berman y DeJong, 2003), de tal manera que la ausencia de frutos favorece el crecimiento del árbol.

El crecimiento en diámetro del tronco fue similar, sin diferencias significativas entre los sistemas de cultivo durante los dos años estudiados, tal y como se puede observar en la Tabla 1, con un valor medio de 4,6 y 6,9 cm en 2018 y 2019, respectivamente.

El tamaño de los árboles durante 2018 fue similar en las plantas cultivadas bajo malla de sombreo y al aire libre, con valores medios de alto, ancho y el volumen de copa de $2,3 \mathrm{~m}, 2,5 \mathrm{~m}$ y $11,4 \mathrm{~m}^{3}$, respectivamente (Tabla 1). Sin embargo, en 2019 se observaron diferencias estadísticamente significativas entre los sistemas de cultivo, de forma que los limeros cultivados bajo malla de sombreo mostraron una mayor altura y volumen de copa, con valores de $2,98 \mathrm{~m}$ y $20,33 \mathrm{~m}^{3}$ respectivamente. El valor del volumen de copa fue un 14 y $17 \%$ mayor que el de las plantas cultivadas al aire libre (en meseta y sin meseta, respectivamente) (Tabla 1), con diferencias estadísticamente significativas desde junio 2019 (Figura 2B). 

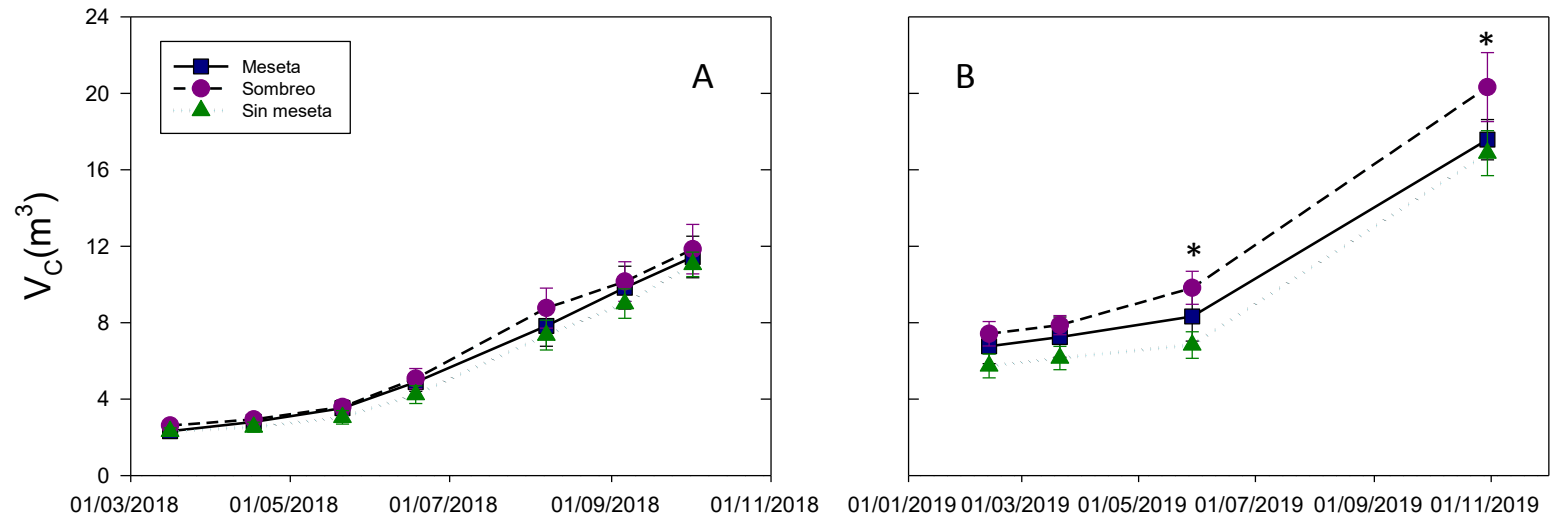

Figura 2. Evolución estacional del volumen de copa $\left(\mathrm{V}_{\mathrm{c}}\right)$ de limeros Bearss en los distintos sistemas de cultivo: Meseta (•), Sombreo (•) y Sin Meseta (A ). 2018 (A) y 2019 (B). Cada punto corresponde a la media de 4 repeticiones \pm error estándar.

En este sentido, Wachsmann et al., (2014) también observaron un volumen de copa mayor en cítricos cultivados bajo malla de sombreo, incrementos que oscilaron entre $6 \mathrm{~m}^{3} \mathrm{y}$ $18 \mathrm{~m}^{3}$ dependiendo del color de la malla, con respecto a los cultivados al aire libre. Una tendencia similar se ha observado en otros cultivos no cítricos como pera (Alaphilippe et al., 2016) o viña (Corvalán et al., 2016). En nuestro ensayo, el mayor volumen de copa fue debido a una mayor $(p<0.001)$ altura de los árboles cultivados bajo malla de sombreo, tal y como apuntan estudios realizados en limón 'Fino 49' (García-Sánchez et al., 2015) y naranja 'Valencia' (Zhou et al., 2018), pues en los cítricos en general se favorece el crecimiento de los brotes en sentido vertical.

Tabla 1. Crecimiento vegetativo: diámetro del tronco $\left(\varnothing_{\text {tronco }}\right)$, alto, ancho y volumen de copa ( $\left.\mathbf{V}_{\text {copa }}\right)$ en limeros jóvenes Bearss en los distintos sistemas de cultivo (2018 y 2019).

\begin{tabular}{ccccc}
\hline Crecimiento & Sistema & $\mathbf{2 0 1 8}$ & $\mathbf{2 0 1 9}$ & $\mathbf{\Delta} \mathbf{2 0 1 8 / 2 0 1 9}$ \\
\hline \multirow{2}{*}{$\boldsymbol{\emptyset}_{\text {tronco }}$} & MESETA & $4,76 \pm 0,17$ & $6,95 \pm 0,24$ & 2,19 \\
$(\mathbf{c m})$ & SOMBREO & $4,57 \pm 0,18$ & $6,80 \pm 0,19$ & 2,23 \\
& SIN MESETA & $4,49 \pm 0,09$ & $6,88 \pm 0,19$ & 2,39 \\
& Significación $(p)$ & $\mathrm{ns}$ & $\mathrm{ns}$ & \\
\hline \multirow{2}{*}{ Alto $_{\mathbf{c}}$} & MESETA & $2,27 \pm 0,08$ & $2,55 \pm 0,13 \mathrm{~b}$ & 0,28 \\
& SOMBREO & $2,33 \pm 0,09$ & $2,98 \pm 0,14 \mathrm{a}$ & 0,65 \\
& SIN MESETA & $2,34 \pm 0,10$ & $2,50 \pm 0,09 \mathrm{~b}$ & 0,16 \\
& Significación $(p)$ & $\mathrm{ns}$ & $* * *$ & \\
\hline \multirow{2}{*}{ Ancho $_{\mathbf{c}}$} & MESETA & $2,54 \pm 0,11$ & $2,97 \pm 0,08$ & 0,43 \\
& SOMBREO & $2,53 \pm 0,10$ & $2,94 \pm 0,13$ & 0,41 \\
& SIN MESETA & $2,46 \pm 0,09$ & $2,93 \pm 0,06$ & 0,47 \\
& Significación $(p)$ & $\mathrm{ns}$ & $\mathrm{ns}$ & \\
\hline & MESETA & $11,44 \pm 1,09$ & $17,58 \pm 1,05 \mathrm{a}$ & 6,14 \\
$\mathbf{V}_{\mathbf{c}}$ & SOMBREO & $11,85 \pm 1,29$ & $20,33 \pm 1,81 \mathrm{~b}$ & 8,48 \\
$\left.\mathbf{m}^{3}\right)$ & SIN MESETA & $11,05 \pm 0,64$ & $16,87 \pm 1,18 \mathrm{a}$ & 5,82 \\
& Significación $(p)$ & $\mathrm{ns}$ & $*$ & \\
\hline
\end{tabular}

Los valores son media de 8 árboles \pm error estándar. Letras diferentes indican diferencias estadísticamente significativas según el test de la $\mathrm{MDS}_{0,05}$. ns, no significativo, ${ }^{* * *} p \leq 0.001$, ${ }^{*} p \leq 0.05$.

\section{1.) Producción}


Los parámetros productivos de las limas en los dos años de estudio se muestran en la Tabla 2. Las plantas cultivadas bajo malla de sombreo mostraron una carga productiva $\left(\mathrm{kg} \mathrm{árbol}^{-1}\right)$ ligeramente mayor a la de las plantas cultivadas al aire libre en ambos ciclos del cultivo ( $\approx 6 \%$ mayor, si bien sin diferencias significativas). El número de frutos por árbol, fue similar en las tres condiciones de cultivo durante ambos años. Sin embargo, el peso unitario del fruto fue mayor en estas plantas en ambos ciclos de cultivo, con diferencias estadísticamente significativas en el año 2019, cuando el peso unitario del fruto fue $16 \mathrm{~g}$ menor en las plantas cultivadas al aire libre. Estos resultados apuntan a que la mayor producción observada en las plantas bajo malla fue resultado de un mayor peso unitario del fruto y no de un mayor número de fruto por árbol. De esta manera, la carga productiva (kg árbol $^{-1}$ ) y el número de frutos por árbol están inversamente relacionados, tal y como indican García-Sánchez et al. (2015) en estudios realizados en limón Fino.

Nuestros resultados coinciden con los obtenidos en estudios realizados en naranja (Zhou et al., 2018) y mandarina (Wachsmann et al., 2014), en los que se observaron aumentos en la producción de las plantas cultivadas en condiciones de sombreo. Este aumento productivo podría ser consecuencia de que la malla de sombreo disminuye la radiación incidente, induciendo un microclima más favorable para la asimilación de $\mathrm{CO}_{2}$ (Medina et al., 2002), aumentando la capacidad fotosintética (Incesu et al., 2016; MiraGarcía et al., 2020) y en definitiva la producción de foto-asimilados necesarios para el óptimo desarrollo del fruto.

El mayor peso unitario (Tabla 2) y diámetro ecuatorial (Figura 3) y longitudinal (datos no mostrados) de las limas cultivadas bajo malla de sombreo puede significar un adelanto en el tamaño comercial de la fruta y en la precocidad de la cosecha, con el consiguiente incremento de su rentabilidad económica.

Tabla 2. Parámetros productivos: $\mathrm{kg}$ árbol ${ }^{-1}$, número de frutos por árbol y peso medio del fruto (g) de limas Bearss en los distintos sistemas de cultivo. 2018 y 2019.

\begin{tabular}{cccc}
\hline Parámetro & Sistema & $\mathbf{2 0 1 8}$ & $\mathbf{2 0 1 9}$ \\
\hline \multirow{2}{*}{ kg árbol $^{-1}$} & MESETA & $12,8 \pm 1,33$ & $34,6 \pm 1,72$ \\
& SOMBREO & $13,4 \pm 0,93$ & $36,8 \pm 1,12$ \\
& SIN MESETA & $12,6 \pm 2,75$ & $35,7 \pm 1,27$ \\
& Significación $(p)$ & $\mathrm{ns}$ & $\mathrm{ns}$ \\
\hline \multirow{2}{*}{$\mathbf{N}^{\mathbf{2}}$ frutos $_{\text {árbol }}^{-1}$} & MESETA & $154 \pm 16,40$ & $481 \pm 23,16$ \\
& SOMBREO & $152 \pm 4,90$ & $419 \pm 20,71$ \\
& SIN MESETA & $160 \pm 4,11$ & $500 \pm 19,51$ \\
& Significación $(p)$ & $\mathrm{ns}$ & $\mathrm{ns}$ \\
\hline \multirow{2}{*}{ Peso medio } & MESETA & $82,93 \pm 1,66$ & $72,11 \pm 0,34 \mathrm{~b}$ \\
& SOMBREO & $88,51 \pm 0,92$ & $88,12 \pm 1,96 \mathrm{a}$ \\
& SIN MESETA & $78,55 \pm 3,75$ & $71,66 \pm 0,92 \mathrm{~b}$ \\
& Significación $(p)$ & $\mathrm{ns}$ & $\star \star *$ \\
\hline
\end{tabular}

Los valores son media de 4 repeticiones \pm error estándar. Letras diferentes indican diferencias estadísticamente significativas según el test de la MDS $_{0,05}$. ${ }^{* * *} p \leq 0.001$, ns: no significativo. 


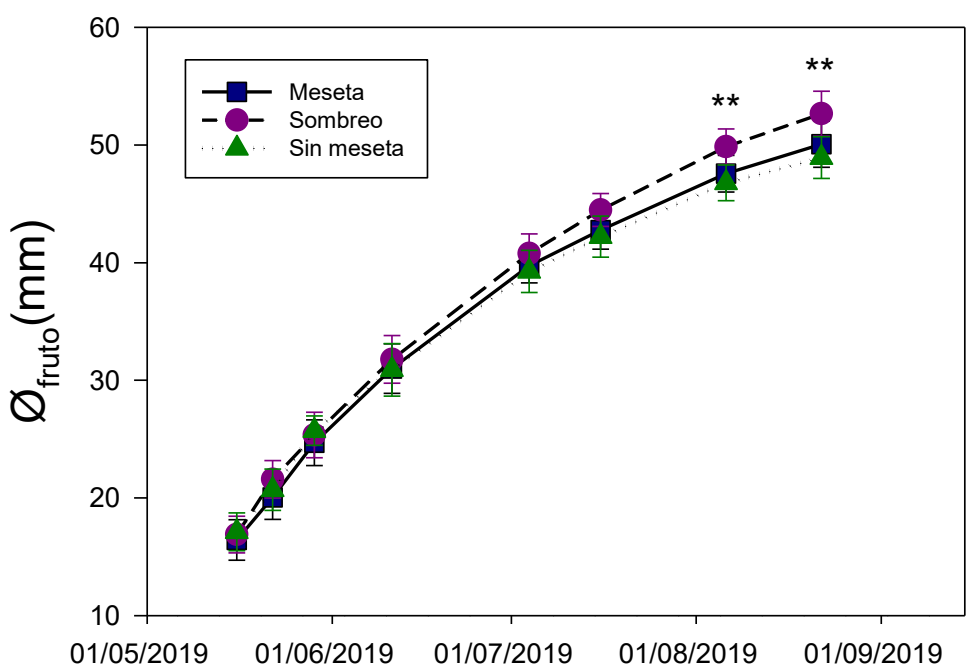

Figura 3. Evolución estacional del crecimiento de las limas Bearss (diámetro ecuatorial, mm)

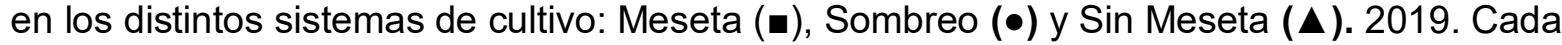
punto corresponde a la media de 4 repeticiones \pm error estándar. ${ }^{* *}$ indica diferencias significativas entre los sistemas de cultivo $p<0,01$.

\section{5) Conclusiones}

El patrón de crecimiento vegetativo (tronco, brotes y copa) de los limeros jóvenes fue similar en los tres sistemas de cultivo estudiados. Sin embargo, los árboles cultivados bajo malla de sombreo mostraron un mayor crecimiento de la copa y una mayor carga productiva y tamaño del fruto que los árboles cultivados al aire libre, tanto en meseta como sin meseta. Estos resultados apuntan a que la disminución de la radiación incidente, provocada por el tipo de malla, crea un microclima en el entorno de los árboles que favorece una mayor asimilación de carbono, promoviendo el crecimiento vegetativo del árbol y la producción de limas de calidad. El carácter juvenil de los árboles requiere continuar el ensayo para corroborar los resultados obtenidos.

\section{6) Agradecimientos}

El trabajo se ha financiado con los proyectos AGL2016-77282-C03-1R y PID2019106226RB-C2-1 (Plan Nacional AEI-Fondos FEDER-UE) y 19903/GERM/15 (Ayudas Grupos de Alto Rendimiento, Fundación Séneca, Región de Murcia).

\section{7) Bibliografía}

Alaphilippe, A., Capowiez, Y., Simon, S., Saudreau, M., Caruso, S., Vergani, S. (2016). Codling moth exclusion netting: an overview of French and Italian experiences. IOBCWPRS Bulletin, 112, 31-35.

Alarcón, J.J., Ortuño, M.F., Nicolás, E., Navarro, A., Torrecillas, A. (2006). Improving wateruse efficiency of young lemon trees by shading with aluminised-plastic nets. Agricultural Water Management, 82, 387-398.

Berman, M.E., DeJong, T.M. (2003). Seasonal patterns of vegetative growth and competition with reproductive sinks in peach (Prunus persica). Journal of Horticultural Science \& Biotechnology, 78, 303-309.

Centro Regional de Estadística de Murcia (CREM) (2019). Estadísticas Agricultura, Ganadería, Selvicultura y Pesca. Evolución de la producción agrícola según tipo de cultivo 2018 [WWW Document]. URL http://econet.carm.es/web/crem/inicio//crem/sicrem/PU590/sec23.html

Consejería de Agua, Agricultura, Ganadería, Pesca y Medioambiente de la Región de 
Murcia (CARM) (2019). Estadística agraria 2018 [WWW Document]. URL https://www.carm.es/web/pagina? IDCONTENIDO=1392\&IDTIPO=100\&RASTRO=c141 $5 \$ \mathrm{~m} 1174$

Corvalán, N., Bastías, R.M., Umanzor, C., Serra, I. (2016). Grapevine root and shoot growth responses to photoselective nets: Preliminary results. Acta Horticulturae, 1136, 89-94.

Food and Agriculture Organization of the United Nations (FAO) (2017). Citrus Fruit: fresh and processed. Statistical Bulletin 2017 [WWW Document]. URL http://www.fao.org/economic/est/est-commodities/citricos/es

García-Sánchez, F., Simón, I., Lidón, V., Manera, F.J., Simón-Grao, S., Pérez-Pérez, J.G., Gimeno, V. (2015). Shade screen increases the vegetative growth but not the production in 'Fino 49' lemon trees grafted on Citrus macrophylla and Citrus aurantium L. Scientia Horticulturae, 194, 175-180.

Hsiao, T.C. (1990). Measurements of plant water status., En: B.A. Stewart and D.R. Nielsen (Eds.), Irrigation of Agricultural Crops (Monograph No. 30). American Society of Agronomy, Madison, pp. 243-279.

Incesu, M., Yeşiloğlu, T., Çimen, B., Yilmaz, B. (2016). Effects of nursery shading on plant growth, chlorophyll content and PSII in 'Lane Late' navel orange seedlings. Acta Horticulturae, 1130, 301-305.

Manja, K., Aoun, M. (2019). The use of nets for tree fruit crops and their impact on the production: A review. Scientia Horticulturae, 246, 110-122.

Mditshwa, A., Magwaza, L.S., Tesfay, S.Z. (2019). Shade netting on subtropical fruit: Effect on environmental conditions, tree physiology and fruit quality. Scientia Horticulturae, 256, 108556.

Medina, C.L., Souza, R.P., Machado, E.C., Ribeiro, R. V., Silva, J.A.B. (2002). Photosynthetic response of citrus grown under reflective aluminized polypropylene shading nets. Scientia Horticulturae, 96, 115-125.

Ministerio de Agricultura Pesca y Alimentación (MAPA) (2018). Anuario de Estadística.

Mira-García, A.B. Conejero, W., Vera, J., Ruiz-Sánchez, M.C. (2020). Leaf water relations in lime trees grown under shade netting and open-air. Plants (MDPI), 9, 510.

Vera, J., Conejero, W., Conesa, M.R., Ruiz-Sánchez, M.C. (2019). Irrigation factor approach based on soil water content: a nectarine orchard case study. Water, 11, 589.

Wachsmann, Y., Zur, N., Shahak, Y., Ratner, K., Giler, Y., Schlizerman, L., Sadka, A., Cohen, S., Garbinshikof, V., Giladi, B., Faintzak, M. (2014). Photoselective anti-hail netting for improved citrus productivity and quality. Acta Horticulturae, 1015, 169-176.

Zhou, K., Jerszurki, D., Sadka, A., Shlizerman, L., Rachmilevitch, S., Ephrath, J. (2018). Effects of photoselective netting on root growth and development of young grafted orange trees under semi-arid climate. Scientia Horticulturae, 238, 272-280. 